\title{
Infections fongiques émergentes
}

\author{
Marcia Garnica Marcio Nucci \\ Hôpital universitaire, Universidade Federal do Rio de Janeiro, Rio de Janeiro, Brésil
}

\section{Mots-clés}

Patients immunodéprimés • Infections fongiques

émergentes

\section{Résumé}

L'épidémiologie des infections fongiques invasives évolue. Des levures autres que Candida albicans et des moisissures autres que Aspergillus fumigatus ont émergé et provoquent des mycoses invasives chez les patients sévèrement immunodéprimés. Ces changements peuvent être liés aux pratiques médicales telles que l'utilisation d'agents antifongiques prophylactiques, mais, dans la majorité des cas, cette évolution est la conséquence de changements chez l'hôte lui-même. Ainsi, le risque et la nature des infections sont influencés par une immunodépression accrue ou différents types d'immunosuppression. Ces facteurs ont modifié l'épidémiologie de l'infection chez les receveurs d'organes, les nouveaux nés prématurés et les patients de réanimation. Notre étude examine l'épidémiologie de certaines infections fongiques qui ont émergé ces dernières années, mettant l'accent sur les facteurs potentiels ayant favorisé leur émergence et sur les conséquences pratiques de ces changements épidémiologiques. De plus, certaines caractéristiques particulières observées chez les enfants et les nouveaux nés sont également présentées.

\section{Introduction}

L'épidémiologie des infections fongiques invasives a changé au cours de ces vingt dernières années. Leur incidence globale a augmenté, de même que la population des patients à risque. Cette dernière comprend maintenant une longue liste de conditions médicales, tels que transplantation d'organes ou de cellules souches hématopoiétiques (CSH), cancer, thérapie immunosuppressive, sida, naissance prématurée, vieillissement et chirurgie lourde [1-4]. De plus, l'étiologie de ces infections a évolué. Dans les années 1980, les levures (en particulier Candida albicans), étaient les principaux agents responsables de mycoses invasives. En revanche, ces dernières années, on observe plus souvent des moisissures que des levures chez certaines catégories de patients, notamment chez les receveurs de CSH. Par ailleurs, chez les patients parmi lesquels la candidose est encore la mycose invasive la plus répandue, Candida albicans n'est responsable que dans moins de 50\% des cas [5]. De même, parmi les moisissures, on rapporte de plus en plus de cas d'aspergillose causée par d'autres espèces que fumigatus [6], telles que des zygomycoses [7], des fusarioses [8], et des phaeohyphomycoses (moisissures noires) [9]. Les raisons exactes de tels changements ne sont pas encore clairement définies. Dans certains cas, il semblerait que certaines pratiques médicales, telles qu'une prophylaxie antifongique [10], et l'utilisation de dispositifs médicaux [11-13] aient influencé ces changements. Néanmoins, dans la plupart

\begin{tabular}{ll}
\hline KARGER & ( 2010 Nestec Ltd., Vevey/S. Karger AG, Basel \\
0250-9644/09/0673-0135\$26.00/0 \\
$\begin{array}{l}\text { Fax +41 61 306 12 34 } \\
\begin{array}{l}\text { E-Mail karger@karger.ch } \\
\text { www.karger.com }\end{array}\end{array}$ & $\begin{array}{l}\text { Accessible en ligne à: } \\
\text { www.karger.com/anf }\end{array}$
\end{tabular}

Dr. Marcio Nucci, Assoc. Prof., Department of Internal Medicine Hematology Service, Hospital Universitário Clementino Fraga Filho Universidade Federal do Rio de Janeiro

Av. Brigadeiro Trompovsky s/n, Rio de Janeiro 21941-590 (Brazil) Fax +55 212562 2460, E-Mail mnucci@ hucff.ufrj.br 
des cas, la raison semble être liée à des changements chez l'hôte lui-même, confronté à des situations d'immunodépression plus sévères [14].

Il existe peu de données sur les infections fongiques émergentes concernant spécifiquement les enfants. Alors que ces infections semblent similaires à celles touchant les adultes par de nombreux aspects, les manifestations épidémiologiques, pathophysiologiques et cliniques sont différentes chez les enfants et les nouveaux nés [15].

Les infections fongiques émergentes représentent un défi majeur pour les cliniciens, avec un mauvais pronostic en raison de différents facteurs: un diagnostic tardif du fait d'un manque de connaissances des cliniciens et des pédiatres sur les manifestations cliniques des nouveaux pathogènes, et le recours obligatoire à un laboratoire compétent pour identifier l'agent de manière fiable et réaliser des tests de sensibilité aux antifongiques, les agents responsables de ces infections émergentes étant généralement résistants à différents antifongiques. Enfin, les patients sévèrement immunodéprimés représentent une population vulnérable pour ces infections émergentes. Dans cette étude nous examinons l'épidémiologie et le diagnostic de certaines maladies fongiques ayant émergé ces dernières années mais également les modifications de l'hôte qui ont contribué à l'apparition de ces infections.

\section{Modifications de l'hôte}

L'hôte peut présenter des facteurs déterminants dans l'émergence d'infections fongiques causées par des pathogènes inhabituels. En général ces champignons ne sont pas pathogènes chez le sujet sain (et même chez les patients peu immunodéprimés) mais ils émergent chez les patients sévèrement immunodéprimés, dont la population s'accroît pour différentes raison.

Chez les patients atteints de cancer, la manière dont la néoplasie sous-jacente est traitée peut avoir une influence significative sur le système immunitaire. Par exemple, l'utilisation de donneurs non-apparentés dans le cadre d'une transplantation de CSH peut induire une leucémie aiguë, une anémie de Fanconi ainsi que d'autres pathologies. Ce type de transplantation est associé à une incidence plus élevée de «graft-versus-host disease» (GVHD, maladie du greffon contre l'hôte ), entraînant un cumul de facteurs favorisant l'immunodépression, notamment au niveau de l'immunité cellulaire (lymphocytes $\mathrm{T}$ ) [16, 17]. Par conséquent, l'aspergillose invasive (AI) est deve- nue une infection émergente chez ces patients. De plus, d'autres sources de cellules souches sont utilisées dans le cadre d'une transplantation de CSH telles que le sang de cordon ombilical, modifiant le cours et la durée de la neutropénie dans la première phase de la transplantation de $\mathrm{CSH}$, et augmentant ainsi le risque d'émergence d'infections fongiques.

Chez les nouveaux nés (population bien connue étant à risque de développer une candidose aiguë invasive), des moisissures ont été observées chez des prématurés pesant moins de 1500 g. L'utilisation de corticoïdes, une hospitalisation prolongée, des cellules phagocytaires immatures et un traumatisme de la peau sont des facteurs de risque majeurs rendant cette population vulnérable. En revanche, la neutropénie n'est pas un facteur de risque important chez les nouveaux nés, comme l'a montré une étude dans laquelle seul 1 bébé sur 44 souffrant d'AI présentait un faible nombre de neutrophiles [18].

De plus en plus de cas de AI ont également été rapportés chez les patients de réanimation, en particulier chez les patients souffrant de bronchopneumopathie chronique obstructive traités par des corticoïdes et chez les patients atteints de graves maladies hépatiques [19, 20].

Plusieurs spécialités pharmaceutiques ont été associées à un risque accru de développer des infections fongiques invasives. On peut citer les analogues de purines telles que la fludarabine, la cladribine et la déoxycoformycine, utilisées dans le traitement des cancers hématologiques [21], et les anticorps monoclonaux. Ainsi, l'alemtuzumab (Campath), un anticorps monoclonal antiCD52, utilisé en cas de malignités lymphoréticulaires, entraîne un épuisement profond des cellules $\mathrm{T}$, de même que l'infliximab (un antagoniste du facteur de nécrose tumorale, TNF- $\alpha$ ) utilisé lors des transplantations de CSH ou d'organes [21-23]. D'autres médicaments sont incriminés, tel que le daclizumab (un antagoniste des récepteurs de l'interleukin-2) utilisé dans le traitement de la GVHD résistante aux stéroïdes chez les patients ayant subi une allogreffe de CSH et pour traiter diverses maladies vasculaires dues au collagène [24-26].

\section{Levures émergentes: la fongémie}

Comme mentionné précédemment, $C$. albicans est considéré comme l'agent le plus souvent responsable des infections invasives causées par les levures. Avec l'introduction et l'utilisation étendue de l'antimycotique fluconazole, les incidences globales de candidémies et d'infec- 
tions par C. albicans ont diminué alors que le nombre d'infections causées par Candida glabrata a augmenté [27]. Ainsi, C. glabrata est considéré, depuis le début des années 1990, comme le deuxième agent le plus fréquemment responsable de candidémie aux États-Unis [28]. De plus, C. glabrata a récemment été associé à une candidose oropharyngée chez des patients traités par radiothérapie pour un cancer de la tête et du cou [29]. Un problème majeur rencontré lors d'une infection par C. glabrata est sa faible sensibilité au fluconazole. En outre, avec les nouveaux dérivés azolés, des multi-résistances se sont développées, le principal mécanisme de résistance au fluconazole étant la surexpression de pompes d'efflux pour plusieurs médicaments [30]. Ainsi, l'analyse de 610 isolats de C. glabrata prélevés sur des patients présentant des infections invasives et vivants dans différentes régions du monde montre que parmi 46 isolats de C. glabrata résistants au fluconazole, seulement $13 \%$ sont sensibles au voriconazole, $4 \%$ au posaconazole et $8,7 \%$ au ravuconazole [31]. Par ailleurs, une série de cas d'infections aiguës dans un seul centre chez des patients traités avec du voriconazole a mis en évidence la possibilité que le C. glabrata résistant au voriconazole devienne une menace pour ces patients [32].

Dans certaines régions du monde, particulièrement en Amérique latine, la deuxième cause majeure de candidémie n'est pas C. glabrata mais Candida parapsilosis ou Candida tropicalis [33], tant chez les adultes que chez les enfants, avec des prévalences de $24-31 \%$ de C. parapsilosis et $16-25 \%$ de $C$. tropicalis [34, 35]. Bien que les raisons de l'émergence de ces organismes ne soient pas connues, certains facteurs sont incriminés: mauvais entretien des cathéters intraveineux, contamination des solutions administrées par voie intraveineuse, et manque d'hygiène (contamination) du personnel de santé. Ces facteurs seraient davantage impliqués dans les infections par C. parapsilosis que la pression de sélection induite par les médicaments [36]. Selon la localisation géographique, différentes espèces de Candida apparaissent comme les pathogènes prédominants. Ceci donne une idée de la complexité épidémiologique. De multiples facteurs tels que les spécialités des centres médicaux régionaux, les pratiques habituelles locales, ainsi que les facteurs propres aux hôtes seraient responsables de cette épidémiologie complexe. Des données préliminaires portant sur des tests in vitro de sensibilité aux échinocandines suggèrent que certains isolats de C. parapsilosis sont moins sensibles que d'autres espèces de Candida [37]. Les conséquences cliniques de ces découvertes ne sont pas connues. Cependant, les échinocandines étant de plus en plus utilisés dans la thérapie primaire des candidémies, le développement de multi-résistances à ces agents pose un réel problème [38].

Candida rugosa a rarement été rapporté comme pathogène humain. En 1994 plusieurs cas de fongémie due à cet organisme ont été observés dans une unité de soins de brûlés aux États-Unis. Aucune source ne fut véritablement identifiée et Dube et al. [39] ont associé cette émergence à l'utilisation de la nystatine en application locale sur les brûlures. Plus récemment, une autre série de cas fut observée dans l'unité de soins intensifs d'un hôpital brésilien au cours d'une étude portant sur la surveillance des candidémies dans 6 institutions situées dans 3 villes différentes. Six cas furent identifiés en l'espace de 4 mois. Tous les patients avaient été exposés à des facteurs de risque bien identifiés: cathéter veineux central, antibiothérapie, chirurgie et assistance respiratoire. Cependant, encore une fois, aucune source d'infection ne fut identifiée mais le typage ADN a montré que tous les isolats étaient génétiquement apparentés [40]. Dans une étude postérieure prospective réalisée dans la même unité chez 349 patients, des prélèvements ont été effectués sur les sites de colonisation habituels (deux fois par semaine) puis mis en culture pour étudier la valeur prédictive de la colonisation par Candida dans le diagnostic des candidémies [41]. De manière surprenante, 15\% des 1400 échantillons positifs contenaient $C$. rugosa et cette espèce représentait $43,7 \%$ des 32 cas de candidémie (données non publiées). Ainsi, l'infection par C. rugosa n'est pas seulement épidémique, comme habituellement décrit; elle peut également s'avérer endémique dans certaines institutions.

Il est connu depuis la fin des années 1980 que les espèces du genre Trichosporon sont responsables de fongémies et d'infections disséminées. Chez les patients atteints d'un cancer et souffrant de neutropénie, cet organisme cause le plus souvent une infection disséminée [42]. Or, dans un rapport récent émanant d'un centre de cancérologie, la plupart des infections étaient des fongémies liées à la présence d'un cathéter [43]. Les raisons de cette évolution ne sont pas claires mais il est possible que l'utilisation répandue du fluconazole ait joué un rôle, cet agent étant actif in vitro contre les espèces du genre Trichosporon [44]. Plus récemment, des cas de trichosporonoses ont été rapportés chez les nouveaux nés [45]. La plupart des patients étaient prématurés avec un poids moyen à la naissance inférieur à $1000 \mathrm{~g}$. La majorité des cas avaient une infection disséminée et dans plus de $90 \%$ des cas, le champignon a été isolé dans les voies respiratoires. 


\section{Autres levures émergentes}

Les infections à cryptocoques, principalement causées par Cryptococcus neoformans, touchent les patients immunodéprimés dans la plupart des régions du monde. Cependant, une autre espèce de Cryptococcus peut infecter des personnes immunocompétentes. Il s'agit de Cryptococcus gattii ayant une distribution géographique très limitée, la plupart des cas étant localisés en Australie. Une évolution récente concernant cet organisme doit toutefois être notée: il a été reconnu comme responsable d'une infection invasive chez des animaux et des personnes immunocompétentes sur l'île de Vancouver à l'Ouest du Canada $[46,47]$ avec une épidémie touchant plus de 100 personnes en 1999 [46]. C. gattii a donc été retrouvé dans des régions climatiques tempérées alors que l'on croyait que ce champignon ne prospérait qu'en zones tropicales. Les raisons de cette évolution n'ont pas encore été déterminées mais il semblerait que des facteurs environnementaux, tel que le réchauffement climatique, pourraient favoriser la propagation de cet organisme [47]. Des études supplémentaires s'avèrent alors nécessaires pour d'une part déterminer les conséquences cliniques d'une infection par ce champignon et d'autre part, définir l'impact que peuvent avoir les facteurs environnementaux dans l'émergence des champignons pathogènes.

\section{Les moisissures émergentes}

Au cours des dix dernières années, l'épidémiologie des infections par moisissures a largement évoluée. Non seulement l'incidence de l'AI a considérablement augmenté [48], mais il y a de plus en plus d'infections causées par des moisissures telles que les espèces du genre Fusarium et les zygomycètes ayant développé une résistance aux antifongiques couramment utilisés [49]. L'incidence de l'AI a progressé de 357\% depuis 1980 [50, 51]; c'est à présent l'infection fongique invasive la plus fréquente, devançant la candidose $[52,53]$.

La plupart des cas d'AI sont observés chez des patients présentant des malignomes hématologiques (principalement dans les cas de leucémie aiguë myéloïde et de leucémie lymphoblastique aiguë [50-54]) ou chez les receveurs de CSH. Cependant, ces dernières années, de plus en plus de cas d'AI ont été diagnostiqués dans d'autres situations cliniques telles qu'une immunodépression congénitale ou une greffe d'organe solide [50]. Ainsi, une étude multicentrique récente sur 139 patients pédiatriques présentant une AI a montré que $23 \%$ des enfants infectés avaient une immunodépression congénitale ou étaient receveurs d'une greffe d'organe solide. Concernant la population adulte, les patients atteints de myélome ont vu l'incidence d'AI augmenter. Ainsi, une étude réalisée chez des patients ayant reçu une greffe de $\mathrm{CSH}$ a montré que le risque de contracter une AI était 4,5 fois supérieur chez les patients atteints de myélome multiple comparés aux patients ayant une leucémie myéloïde chronique en phase chronique [6]. Le nombre croissant de cas d'AI rapportés chez des patients atteints de myélome multiple peut être dû à des modifications de leur traitement, et notamment à une thérapie agressive et séquentielle avec chimiothérapie intensive, autogreffes et allogreffes non myéloablatives [55].

L'âge a récemment été considéré comme un facteur de risque de l'AI avec une plus forte incidence chez les patients de plus de 10 ans. Ceci s'explique par le fait qu'une colonisation antérieure est une condition nécessaire au développement des infections fongiques invasives [5658].

A présent, une AI apparaît plus tardivement chez les patients ayant subi une allogreffe de CSH et dans le cadre d'un traitement de GVHD par thérapie immunosuppressive (surtout à doses élevées de corticoïdes); elle est parfois diagnostiquée assez tôt après la greffe [6]. Ceci est dû aux modifications des procédures de transplantation ayant entraîné une réduction de la durée de neutropénie et une augmentation de l'incidence et de la sévérité des cas de GVHD: greffes à conditionnement d'intensité réduite (telles que les greffes non-myéloablatives), greffes avec donneurs non apparentés et non compatibles, infusion des lymphocytes du donneur et utilisation d'anticorps monoclonaux tel que infliximab $[23,25]$. La plupart des patients pédiatriques atteints d'AI présentent de nombreux facteurs immunosuppressifs: GVHD dans 30\% des cas, corticothérapie dans $70 \%$ des cas et thérapie immunosuppressive dans $43 \%$ des cas (cyclosporine, tacrolimus, daclizumab) [50]. Une étude prospective récente chez des enfants atteints de cancer a montré que $22 \%$ des infections fongiques invasives sont observées chez les enfants ayant subi une greffe de CSH. Parmi eux, 77\% ont reçu des CSH de donneurs non apparentés et seulement $23 \%$ ont reçu des CSH de donneurs apparentés. En revanche, aucun cas d'infection fongique invasive n'a été observé après une autogreffe [56].

La lymphopénie est un facteur de risque d'AI chez les enfants atteints de cancer et les receveurs de greffes de $\mathrm{CSH}$. La plupart des patients non neutropéniques présentent une lymphopénie [56]. 
L'apparition tardive d'une AI a également été rapportée chez les transplantés hépatiques pour diverses raisons: ils ont une meilleure survie post-greffe, une apparition tardive du cytomégalovirus, et un plus grand nombre de patients présentent un dysfonctionnement tardif de l'allogreffe [59].

Une autre tendance a été observée concernant l'AI: on rapporte de plus en plus de cas d'infection causée par des espèces autre que $A$. fumigatus, principalement chez les enfants, et pour plus de $50 \%$ des cas $[15,60,61]$. Ainsi, Aspergillus flavus est l'espèce la plus fréquente et l'infection est cutanée [50,60,62,63]. Une autre espèce, Aspergillus terreus est particulièrement inquiétante car elle est résistante, in vitro, à l'amphotéricine $\mathrm{B}$ et sa réponse clinique à cet antifongique est faible [44]. Une étude sur l'incidence d'AI causée par $A$. terreus dans un hôpital universitaire a ainsi montré une augmentation de l'incidence de $2,1 \%$ en 1996 à 10,2\% en 2001 [64], les raisons de cette émergence étant inconnues. De plus, ce phénomène est très peu décrit chez les enfants. L'AI causée par $A$. terreus présente un faible pronostic avec un taux de mortalité global de $66 \%$. Un traitement au voriconazole semblerait cependant donner de meilleurs résultats [65].

La manifestation clinique de l'aspergillose chez les enfants est similaire à celle des adultes, les poumons étant impliqués dans la grande majorité des cas. Néanmoins, l'image radiologique peut être différente: cavités et nodules présents dans seulement $25 \%$ des cas sans aucun signe de formation de croissant gazeux [66] ou cavités et croissant gazeux observés seulement chez les enfants plus âgés [67]. L’aspergillose cutanée a été plus souvent rapportée chez les enfants que chez les adultes, en tant qu'infection soit localisée (incidence de 5 à 14\%) soit disséminée $[50,68]$.

Une autre différence importante entre les adultes et les enfants est la pertinence d'un dosage sérique du galactomannane comme test ancillaire servant à établir le diagnostic de l'AI [69]. Ce dosage, par une réaction immunoenzymatique de type sandwich, est disponible depuis 1997 et permet de détecter l'antigène aspergillaire. Ce test peut être utilisé comme outil diagnostique, en plus d'autres outils, pour identifier les patients à haut risque de développer une aspergillose (en particulier les patients atteints de leucémie aiguë et les greffés de CSH) ou pour contrôler la réponse au traitement [70, 71]. Plusieurs études ont évalué le dosage du galactomannane chez les enfants. Les premières rapportaient des taux élevés de faux-positifs et ne recommandaient pas ce test $[15,72]$. Des études plus récentes ont quant à elles rapporté des valeurs prédictives négatives similaires à celles observées chez les adultes [57, 73, 74].

La zygomycose est devenue une infection importante chez les patients sévèrement immunodéprimés. Une étude multicentrique sur les infections fongiques chez les transplantés a révélé une augmentation de l'incidence de zygomycose de $4 \%$ en 2001 à $25 \%$ en 2003 . Ainsi, entre mars 2001 et avril 2002, sur 4358 greffés de CSH, 4 $(0,09 \%)$ ont développé une zygomycose alors qu'entre mai 2002 et juin 2003, sur 6660 greffés, 11 cas $(0,17 \%)$ de zygomycose ont été rapportés [75]. L'analyse multifactorielle des facteurs associés à l'émergence de la zygomycose, en comparaison avec l'AI, a révélé qu'une exposition antérieure au voriconazole représentait un facteur de risque pour les greffés de CSH (odds ratio de 7,7) et d'organe solide (odds ratio de 10,7). Ceci est supporté par 4 cas de zygomycose aiguë rapportés chez 15 patients sous voriconazole [32, 76-78] et par une étude monocentrique comparative dont l'analyse multifactorielle montre également qu'une exposition préalable au voriconazole est un facteur de risque de zygomycose [79]. Cependant, même si l'utilisation du voriconazole a probablement contribué à l'émergence de zygomycoses, d'autres facteurs, tels que la gravité et la nature des immunodépressions, peuvent s'avérer aussi importants. Certaines données indiquent d'ailleurs que l'incidence croissante de zygomycose est antérieure à l'introduction du voriconazole en pratique clinique [7]. Une autre explication possible à cette augmentation de l'incidence de zygomatose est la diminution potentielle, ces dernières années, de la mortalité liée à l'AI permettant aux patients de vivre assez longtemps pour développer ensuite une infection accompagnée d'une immunodépression persistante. La zygomycose peut alors être considérée comme la troisième "menace» d'infections fongiques chez les receveurs d'allogreffes de $\mathrm{CSH}$.

La plupart des cas de zygomycose chez les patients pédiatriques concernent les enfants atteints de cancer pendant la période de neutropénie, les prématurés et les patients diabétiques. La mortalité est élevée malgré une thérapie antifongique associée ou non à la chirurgie [80].

De plus en plus de cas de fusariose ont été rapportés chez des patients immunodéprimés, tels que chez les patients ayant subi une transplantation de CSH. Ainsi, une étude multicentrique rétrospective [8] indique que l'immunodépression, et plus précisément un déficit sévère de l'immunité cellulaire, semble être la cause principale de cette augmentation. En effet, l'incidence de fusariose était plus élevée après des greffes de donneurs incompatibles et la plupart de ces patients présentaient soit une neutropénie sévère, soit une GVHD. Un nombre impor- 
tant de ces cas a été diagnostiqué plus d'un an après la transplantation et ceux-là n'étaient pas neutropéniques.

En plus de ces infections, une longue liste d'autres champignons, comprenant les espèces des genres Scedosporium, Acremonium, et Phialemonium, serait ponctuellement responsable d'infections chez les patients immunodéprimés. Ainsi, une augmentation de l'incidence des infections à moisissures non aspergillaires a été observée chez les transplantés hépatiques [81] et les receveurs de greffes de CSH [48], probablement due à une immunodépression encore plus sévère. Il est intéressant de noter que beaucoup de ces organismes semblent avoir une localisation géographique restreinte. Par exemple, les infections à Fusarium ont été surtout rapportées dans les centres de soin du sud des États-Unis et en Amérique latine [8] alors que Scedosporium est responsable d'infections en Espagne [82]. Des études sont maintenant requises pour déterminer si cette restriction géographique reflète un biais de notification, une réelle répartition des hôtes à risque ou une influence environnementale sur la prévalence fongique.

\section{Conclusion}

L'émergence de pathogènes fongiques est la conséquence d'une interaction dynamique entre l'hôte, l'environnement et le pathogène. Bien que certaines infections fongiques semblent avoir émergé suite à la pression sélective résultant de l'utilisation étendue d'agents antifongiques prophylactiques (telle que l'infection causée par C. glabrata), la grande majorité émerge dans les situations d'immunodépression sévère. Par conséquent, comme le nombre et les populations de patients immunodéprimés augmentent, il n'est pas surprenant que la liste des infections fongiques s'allonge. Les cliniciens et les pédiatres doivent avoir conscience de l'existence de ces nouveaux pathogènes et connaître les maladies qu'ils causent et leurs manifestations cliniques afin d'établir rapidement le bon diagnostic.

\section{Remerciement}

Dr. Marcio Nucci a été financé par un contrat $\mathrm{CNPq}$ 300235/93-3.

\section{Bibliographie}

1 Brown JM: Fungal infections in bone marrow transplant patients. Curr Opin Infect Dis 2004; 17:347-352.

2 Fishman JA: Overview: fungal infections in the transplant patient. Transpl Infect Dis 2002;4(suppl 3):3-11.

-3 Kauffman CA: Fungal infections in olde adults. Clin Infect Dis 2001;33:550-555.

4 Kaufman D: Fungal infection in the very low birthweight infant. Curr Opin Infect Dis 2004;17:253-259.

5 Pappas PG, Rex JH, Lee J, et al: A prospective observational study of candidemia: epidemiology, therapy, and influences on mortality in hospitalized adult and pediatric patients. Clin Infect Dis 2003;37:634-643.

6 Marr KA, Carter RA, Boeckh M, et al: Invasive aspergillosis in allogeneic stem cell transplant recipients: changes in epidemiology and risk factors. Blood 2002;100:43584366.

7 Kauffman CA: Zygomycosis: reemergence of an old pathogen. Clin Infect Dis 2004;39: 588-590.

8 Nucci M, Marr KA, Queiroz-Telles F, et al: Fusarium infection in hematopoietic stem cell transplant recipients. Clin Infect Dis 2004;38:1237-1242.

-9 Revankar SG, Patterson JE, Sutton DA, et al: Disseminated phaeohyphomycosis: review of an emerging mycosis. Clin Infect Dis 2002;34:467-476.
10 Snydman DR: Shifting patterns in the epidemiology of nosocomial Candida infections. Chest 2003; 123:500S-503S.

11 Hsueh PR, Teng LJ, Ho SW, Luh KT: Catheter-related sepsis due to Rhodotorula glutinis. J Clin Microbiol 2003;41:857-859.

12 Reinhardt JF, Ruane PJ, Walker LJ, George WL: Intravenous catheter-associated fungemia due to Candida rugosa. J Clin Microbiol 1985;22:1056-1057.

13 Yamada S, Maruoka T, Nagai K, et al: Catheter-related infections by Hansenula anomala in children. Scand J Infect Dis 1995;27: 85-87.

14 Procop GW, Roberts GD: Emerging fungal diseases: the importance of the host. Clin Lab Med 2004;24:691-719.

15 Steinbach WJ: Pediatric aspergillosis: disease and treatment differences in children. Pediatr Infect Dis J 2005;24:358-364.

16 Schutt P, Brandhorst D, Stellberg W, et al: Immune parameters in multiple myeloma patients: influence of treatment and correlation with opportunistic infections. Leuk Lymphoma 2006;47:1570-1582.

17 Wingard JR: Opportunistic infections after blood and marrow transplantation. Transpl Infect Dis 1999;1:3-20.
8 Groll AH, Jaeger G, Allendorf A, et al: Invasive pulmonary aspergillosis in a critically ill neonate: case report and review of invasive aspergillosis during the first 3 months of life. Clin Infect Dis 1998;27:437-452.

19 Bulpa P, Dive A, Sibille Y: Invasive pulmonary aspergillosis in patients with chronic obstructive pulmonary disease. Eur Respir J 2007;30:782-800.

20 Meersseman W, Lagrou K, Maertens J, Van Wijngaerden E: Invasive aspergillosis in the intensive care unit. Clin Infect Dis 2007;45: 205-216.

21 Anaissie EJ, Kontoyiannis DP, O’Brien S, et al: Infections in patients with chronic lymphocytic leukemia treated with fludarabine. Ann Intern Med 1998;129:559-566.

22 Keating MJ, Flinn I, Jain V, et al: Therapeutic role of alemtuzumab (Campath-1H) in patients who have failed fludarabine: results of a large international study. Blood 2002;99: 3554-3561.

23 Nath DS, Kandaswamy R, Gruessner R, et al: Fungal infections in transplant recipients receiving alemtuzumab. Transplant Proc 2005;37:934-936.

24 Labbe AC, Su SH, Laverdiere M, et al: High incidence of invasive aspergillosis associated with intestinal graft-versus-host disease following nonmyeloablative transplantation. Biol Blood Marrow Transplant 2007; 13:1192-1200. 
25 Marty FM, Lee SJ, Fahey MM, et al: Infliximab use in patients with severe graft-versus-host disease and other emerging risk factors of non-Candida invasive fungal infections in allogeneic hematopoietic stem cell transplant recipients: a cohort study. Blood 2003; 102:2768-2776.

-26 Tsiodras S, Samonis G, Boumpas DT, Kontoyiannis DP: Fungal infections complicating tumor necrosis factor alpha blockade therapy. Mayo Clin Proc 2008;83:181-194.

27 Trick WE, Fridkin SK, Edwards JR, et al: Secular trend of hospital-acquired candidemia among intensive care unit patients in the United States during 1989-1999. Clin Infect Dis 2002;35:627-630.

28 Pfaller MA, Diekema DJ: Twelve years of fluconazole in clinical practice: global trends in species distribution and fluconazole susceptibility of bloodstream isolates of Candida. Clin Microbiol Infect 2004;10(suppl 1):1123.

-29 Redding SW, Dahiya MC, Kirkpatrick WR, et al: Candida glabrata is an emerging cause of oropharyngeal candidiasis in patients receiving radiation for head and neck cancer. Oral Surg Oral Med Oral Pathol Oral Radiol Endod 2004;97:47-52.

-30 Bennett JE, Izumikawa K, Marr KA: Mechanism of increased fluconazole resistance in Candida glabrata during prophylaxis. Antimicrob Agents Chemother 2004;48:17731777.

-31 Pfaller MA, Messer SA, Boyken L, et al: Geographic variation in the susceptibilities of invasive isolates of Candida glabrata to seven systemically active antifungal agents: a global assessment from the ARTEMIS Antifungal Surveillance Program conducted in 2001 and 2002. J Clin Microbiol 2004;42: 3142-3146.

- 32 Imhof A, Balajee SA, Fredricks DN, et al: Breakthrough fungal infections in stem cell transplant recipients receiving voriconazole. Clin Infect Dis 2004;39:743-746.

- 33 Colombo AL, Nucci M, Salomao R, et al: High rate of non-albicans candidemia in Brazilian tertiary care hospitals. Diagn Microbiol Infect Dis 1999;34:281-286.

-34 Pasqualotto AC, de Moraes AB, Zanini RR, Severo LC: Analysis of independent risk factors for death among pediatric patients with candidemia and a central venous catheter in place. Infect Control Hosp Epidemiol 2007; 28:799-804.

- 35 Stamos JK, Rowley AH: Candidemia in a pediatric population. Clin Infect Dis 1995;20: 571-575.

- 36 Pfaller MA: Nosocomial candidiasis: emerging species, reservoirs, and modes of transmission. Clin Infect Dis 1996;22(suppl 2):S89-S94.
37 Pfaller MA, Diekema DJ, Jones RN, et al: Trends in antifungal susceptibility of Candida spp. isolated from pediatric and adult patients with bloodstream infections: SENTRY Antimicrobial Surveillance Program, 1997 to 2000. J Clin Microbiol 2002;40:852856.

38 Moudgal V, Little T, Boikov D, Vazquez JA: Multiechinocandin- and multiazole-resistant Candida parapsilosis isolates serially obtained during therapy for prosthetic valve endocarditis. Antimicrob Agents Chemother 2005;49:767-769.

39 Dube MP, Heseltine PN, Rinaldi MG, et al: Fungemia and colonization with nystatinresistant Candida rugosa in a burn unit. Clin Infect Dis 1994;18:77-82.

40 Colombo AL, Melo AS, Crespo Rosas RF, et al: Outbreak of Candida rugosa candidemia: an emerging pathogen that may be refractory to amphotericin B therapy. Diagn Microbiol Infect Dis 2003;46:253-257.

41 Rosas R, Nucci M, Castelo A, et al: Predictive value of Candida spp. colonization in the diagnosis of candidemia in intensive care unit patients (abstract M-269). Program and Abstracts 44th Interscience Conference on Antimicrobial Agents and Chemotherapy (Washington). Washington, American Society for Microbiology, 2004, p 410.

42 Walsh TJ, Newman KR, Moody M, et al: Trichosporonosis in patients with neoplastic disease. Medicine (Baltimore) 1986;65:268279.

43 Kontoyiannis DP, Torres HA, Chagua M, et al: Trichosporonosis in a tertiary care cancer center: risk factors, changing spectrum and determinants of outcome. Scand J Infect Dis 2004;36:564-569.

44 Pfaller MA, Diekema DJ: Rare and emerging opportunistic fungal pathogens: concern for resistance beyond Candida albicans and Aspergillus fumigatus. J Clin Microbiol 2004; 42:4419-4431.

45 Salazar GE, Campbell JR: Trichosporonosis, an unusual fungal infection in neonates. Pediatr Infect Dis J 2002;21:161-165.

46 Hoang LM, Maguire JA, Doyle P, et al: Cryptococcus neoformans infections at Vancouver Hospital and Health Sciences Centre (1997-2002): epidemiology, microbiology and histopathology. J Med Microbiol 2004; 53:935-940.

47 Kidd SE, Hagen F, Tscharke RL, et al: A rare genotype of Cryptococcus gattii caused the cryptococcosis outbreak on Vancouver Island (British Columbia, Canada). Proc Natl Acad Sci USA 2004;101:17258-17263.

48 Marr KA, Carter RA, Crippa F, et al: Epidemiology and outcome of mould infections in hematopoietic stem cell transplant recipients. Clin Infect Dis 2002;34:909-917.

49 Nucci M: Emerging moulds: Fusarium, Scedosporium and Zygomycetes in transplant recipients. Curr Opin Infect Dis 2003;16: 607-612.
50 Burgos A, Zaoutis TE, Dvorak CC, et al: Pediatric invasive aspergillosis: a multicenter retrospective analysis of 139 contemporary cases. Pediatrics 2008;121:e1286-e1294.

51 McNeil MM, Nash SL, Hajjeh RA, et al: Trends in mortality due to invasive mycotic diseases in the United States, 1980-1997. Clin Infect Dis 2001;33:641-647.

52 Groll AH, Shah PM, Mentzel C, et al: Trends in the postmortem epidemiology of invasive fungal infections at a university hospital. J Infect 1996;33:23-32.

53 van Burik JH, Leisenring W, Myerson D, et al: The effect of prophylactic fluconazole on the clinical spectrum of fungal diseases in bone marrow transplant recipients with special attention to hepatic candidiasis. An autopsy study of 355 patients. Medicine (Baltimore) 1998;77:246-254.

54 Cornet M, Fleury L, Maslo C, et al: Epidemiology of invasive aspergillosis in France: a six-year multicentric survey in the Greater Paris area. J Hosp Infect 2002;51:288-296.

55 Barlogie B, Shaughnessy J, Tricot G, et al: Treatment of multiple myeloma. Blood 2004;103:20-32.

56 Castagnola E, Cesaro S, Giacchino M, et al: Fungal infections in children with cancer: a prospective, multicenter surveillance study. Pediatr Infect Dis J 2006;25:634-639.

57 Castagnola E, Faraci M, Moroni C, et al: Invasive mycoses in children receiving hemopoietic SCT. Bone Marrow Transplant 2008; 41(suppl 2):S107-S111.

58 Dvorak CC, Steinbach WJ, Brown JM, Agarwal R: Risks and outcomes of invasive fungal infections in pediatric patients undergoing allogeneic hematopoietic cell transplantation. Bone Marrow Transplant 2005;36:621629.

59 Singh N, Avery RK, Munoz P, et al: Trends in risk profiles for and mortality associated with invasive aspergillosis among liver transplant recipients. Clin Infect Dis 2003; 36:46-52.

60 Herbrecht R, Auvrignon A, Andres E, et al: Efficacy of amphotericin B lipid complex in the treatment of invasive fungal infections in immunosuppressed paediatric patients. Eur J Clin Microbiol Infect Dis 2001;20:7782.

61 Walsh TJ, Lutsar I, Driscoll T, et al: Voriconazole in the treatment of aspergillosis, scedosporiosis and other invasive fungal infections in children. Pediatr Infect Dis J 2002; 21:240-248.

62 Abbasi S, Shenep JL, Hughes WT, Flynn PM: Aspergillosis in children with cancer: a 34year experience. Clin Infect Dis 1999;29: 1210-1219.

63 Walmsley S, Devi S, King S, et al: Invasive Aspergillus infections in a pediatric hospital: a ten-year review. Pediatr Infect Dis J 1993;12:673-682. 
64 Baddley JW, Pappas PG, Smith AC, Moser SA: Epidemiology of Aspergillus terreus at a university hospital. J Clin Microbiol 2003; 41:5525-5529.

-65 Steinbach WJ, Benjamin DK Jr, Kontoyiannis DP, et al: Infections due to Aspergillus terreus: a multicenter retrospective analysis of 83 cases. Clin Infect Dis 2004;39:192-198.

-66 Thomas KE, Owens CM, Veys PA, et al: The radiological spectrum of invasive aspergillosis in children: a 10-year review. Pediatr Radiol 2003;33:453-460.

-67 Taccone A, Occhi M, Garaventa A, et al: CT of invasive pulmonary aspergillosis in children with cancer. Pediatr Radiol 1993;23: 177-180.

68 Vonberg RP, Gastmeier P: Nosocomial aspergillosis in outbreak settings. J Hosp Infect 2006;63:246-254.

69 De Pauw B, Walsh TJ, Donnelly JP, et al: Revised definitions of invasive fungal disease from the European Organization for Research and Treatment of Cancer/Invasive Fungal Infections Cooperative Group and the National Institute of Allergy and Infectious Diseases Mycoses Study Group (EORTC/MSG) Consensus Group. Clin Infect Dis 2008;46:1813-1821.

-70 Miceli MH, Grazziutti ML, Woods G, et al: Strong correlation between serum aspergillus galactomannan index and outcome of aspergillosis in patients with hematological cancer: clinical and research implications. Clin Infect Dis 2008;46:1412-1422.
71 Woods G, Miceli MH, Grazziutti ML, et al: Serum Aspergillus galactomannan antigen values strongly correlate with outcome of invasive aspergillosis: a study of 56 patients with hematologic cancer. Cancer 2007;110: 830-834.

72 Sulahian A, Tabouret M, Ribaud P, et al: Comparison of an enzyme immunoassay and latex agglutination test for detection of galactomannan in the diagnosis of invasive aspergillosis. Eur J Clin Microbiol Infect Dis 1996;15:139-145.

73 Steinbach WJ, Addison RM, McLaughlin L, et al: Prospective Aspergillus galactomannan antigen testing in pediatric hematopoietic stem cell transplant recipients. Pediatr Infect Dis J 2007;26:558-564.

74 Sulahian A, Boutboul F, Ribaud P, et al: Value of antigen detection using an enzyme immunoassay in the diagnosis and prediction of invasive aspergillosis in two adult and pediatric hematology units during a 4-year prospective study. Cancer 2001;91:311-318.

75 Park BJ, Kontoyiannis DP, Pappas PG, et al: Comparison of zygomycosis and fusariosis to invasive aspergillosis among transplant recipients reporting to TRANSNET (abstract M-666). Program and Abstracts 44th Interscience Conference on Antimicrobial Agents and Chemotherapy (Washington), Washington, American Society for Microbiology, 2004, p 411.
76 Kobayashi K, Kami M, Murashige N, et al: Breakthrough zygomycosis during voriconazole treatment for invasive aspergillosis. Haematologica 2004;89:ECR42.

77 Marty FM, Cosimi LA, Baden LR: Breakthrough zygomycosis after voriconazole treatment in recipients of hematopoietic stem-cell transplants. N Engl J Med 2004; 350:950-952.

78 Siwek GT, Dodgson KJ, Magalhaes-Silverman $\mathrm{M}$, et al: Invasive zygomycosis in hematopoietic stem cell transplant recipients receiving voriconazole prophylaxis. Clin Infect Dis 2004;39:584-587.

79 Kontoyiannis DP, Lionakis MS, Lewis RE, et al: Zygomycosis in a tertiary-care cancer center in the era of Aspergillus-active antifungal therapy: a case-control observational study of 27 recent cases. J Infect Dis 2005; 191:1350-1360.

80 Zaoutis TE, Roilides E, Chiou CC, et al: Zygomycosis in children: a systematic review and analysis of reported cases. Pediatr Infect Dis J 2007;26:723-727.

81 Husain S, Alexander BD, Munoz P, et al: Opportunistic mycelial fungal infections in organ transplant recipients: emerging importance of non-Aspergillus mycelial fungi. Clin Infect Dis 2003;37:221-229.

82 Bouza E, Munoz P: Invasive infections caused by Blastoschizomyces capitatus and Scedosporium spp. Clin Microbiol Infect 2004;10(suppl 1):76-85. 
